\title{
Prevalence and factors associated with mild cognitive impairment on screening in older Malaysians
}

\begin{abstract}
Objective: This study was aimed to determine the prevalence and predictors of mild cognitive impairments (MCIs) in Malaysia. Method: This is a cross-sectional community-based study among elderlies aged 60 years and above in the four states of Malaysia - Perak, Kelantan, Selangor and Johor. A multi-stage stratified random sampling method was used for the data collection. Mini Mental State Examination score ?22 was used to diagnosed MCI and analyzed by using multivariate logistic regression. Results: A total of 2112 participants were recruited into this study. Mean age of the participants was $69.2 \pm 18.8$ years, and $51.4 \%$ of them were females. The ethnic distribution was $63.4 \%$ Malaysian, 31.4\% Chinese, 5.0\% Indians, and $0.2 \%$ others. The overall prevalence of MCI was $68 \%(n=1436 / 2112)$. In multivariate logistic regression analysis, participant who were older (OR 1.02, 95\% CI 1.001.04), did not receive formal education (OR 6.85, 95\% CI 4.58-10.25), had primary education (OR 2.35 95\% CI 1.84-3.00), were lonely (OR 1.18 95\% CI 1.05-1.32), and had low level of life satisfaction (OR 1.68 95\% CI 1.02-2.67), had significant positive correlations with MCI. Conclusion: MCI is present in seven out of ten elderly Malaysians. Aging population, presence of low education level, loneliness and low life satisfaction level seem to facilitate development of MCI.
\end{abstract}

Keyword: Aging; Cognitive impairment; Dementia; Elderly; Malaysia; Prevalence 